\title{
A Radiomics nomogram for preoperative predicting recurrence of low grade gliomas based on multiparametric MRI
}

\author{
Zhenhua Wang (D 57310537@qq.com) \\ The Second Affiliated Hosptial of Nanchang University \\ Xinlan Xiao \\ Nanchang University Second Affiliated Hospital \\ Zhaotao Zhang \\ Nanchang University Second Affiliated Hospital \\ Keng He \\ Nanchang University Second Affiliated Hospital \\ Peipei Pang \\ GE Healthcare Life Sciences \\ Feng $\mathrm{Hu}$ \\ Nanchang University Second Affiliated Hospital
}

\section{Research Article}

Keywords: low grade glioma, recurrence, nomogram

Posted Date: March 22nd, 2021

DOI: https://doi.org/10.21203/rs.3.rs-345383/v1

License: (c) (i) This work is licensed under a Creative Commons Attribution 4.0 International License. Read Full License 


\section{Abstract}

Objective To develop a radiomics nomogram to predict the recurrence of Low grade glioma(LGG) after their first surgery; Methods A retrospective analysis of pathological, clinical and Magnetic resonance image(MRI) of LGG patients who underwent surgery and had a recurrence between 2017 and 2020 in our hospital was performed. After a rigorous selection,64 patients were eligible and enrolled in the study(22 cases were with recurrent gliomas), which was randomly assigned in a 7:3 ratio to either the training set and validation set; T1WI,T2WI fluid-attenuated-inversion-recovery(T2WIFLAIR) and contrast-enhanced T1-weighted(T1CE) sequences, 396 radiomics features were extracted from each image sequence, minimum-redundancy maximum-relevancy (mRMR) alone or combining with univariate logistic analysis were used for features screening, the screened features were performed by multivariate logistic regression analysis and developed a predictive model both in training set and validation set; Receiver operating characteristic(ROC) curve, calibration curve, and decision curve analysis(DCA) were used to assess the performance of each model. Results The radiomics nomogram derived from three MRI sequence yielded an ideal performance than the individual ones, the AUC in the training set and validation set were 0.966 and 0.93 respectively, $95 \%$ confidence interval $(95 \% \mathrm{Cl})$ were $0.949-0.99$ and $0.905-0.973$ respectively; the calibration curves indicated good agreement between the predictive and the actual probability. The DCA demonstrated that a combination of three sequences had more favorable clinical predictive value than single sequence imaging. Conclusion Our multiparametric radiomics nomogram could be an efficient and accurate tool for predicting the recurrence of LGG after its first resection.

\section{Introduction}

Glioma is a common malignant tumor originating from central glial cells and has devastating effects[1-4], LGG have long been considered to be a benign subgroup of clinical biological behaviors in brain gliomas,and there is growing evidence demonstrate that it is also a class II glioma[World Health Organization(WHO) 2016 classification], but its biological behavior and clinical prognosis are far different.Although there are many treatment options for glioma, their therapeutic efficacy is still unsatisfactory[5-7],the same therapy strategy,some patient with LGG after a tumor resection without recurrence but some one was found recurrence soon,so some patients have long-term OS or long-term FPS, some patients showed highly malignant outcome.Although in many previous studies, there are some factors like age,the extent resection, expression of some specific genes et al that will affect the clinical outcome[8],however,so far,there is not exist a quantitative and accurate tool to evaluate the risk of recurrence after their first surgery,so our group want develop a radiomics nomogram tool to predict the recurrent possibility.

Table 1. RANO criteria for evaluating the efficacy of low-grade gliomas after treatment

\begin{tabular}{|c|c|c|c|c|}
\hline Criterion & $\begin{array}{l}\text { Complete } \\
\text { remission }\end{array}$ & Partial remission & Stable disease & Progress disease \\
\hline $\mathrm{T} 1+\mathrm{CE}$ & Not seen & Decrease $\geq 50 \%$ & $\begin{array}{l}\text { Increase or decrease in the range } \\
\text { of }-25 \% \sim+25 \%\end{array}$ & Increase $\geq 25 \% \star$ \\
\hline T2/FIAIR & $\begin{array}{l}\text { Stable or } \\
\text { diminished }\end{array}$ & $\begin{array}{l}\text { Stable or } \\
\text { diminished }\end{array}$ & Stable or diminished & Increase* \\
\hline New lesion & $\begin{array}{l}\text { None(apart from } \\
\text { those } \\
\text { consistent with } \\
\text { radiation } \\
\text { effects, and no } \\
\text { new or } \\
\text { increased } \\
\text { enhancement) }\end{array}$ & $\begin{array}{l}\text { None(apart from } \\
\text { those } \\
\text { consistent with } \\
\text { radiation } \\
\text { effects, and no } \\
\text { new or } \\
\text { increased } \\
\text { enhancement) }\end{array}$ & $\begin{array}{l}\text { None(apart from those } \\
\text { consistent with radiation } \\
\text { effects, and no new or } \\
\text { increased enhancement) }\end{array}$ & Present* \\
\hline Corticosteroids & None & $\begin{array}{l}\text { Stable or } \\
\text { diminished }\end{array}$ & Stable or diminished & Not apply \\
\hline Clinical status & $\begin{array}{l}\text { Stable or } \\
\text { improve }\end{array}$ & $\begin{array}{l}\text { Stable or } \\
\text { improve }\end{array}$ & Stable or improve & $\begin{array}{l}\text { Deteriorate*(not attributable to } \\
\text { other causes apart from the tumor, or decrease in } \\
\text { corticosteroid dose) }\end{array}$ \\
\hline $\begin{array}{l}\text { Requirement for } \\
\text { response }\end{array}$ & All & All & All & Any \\
\hline
\end{tabular}

Abbreviation:CE:contrast enhanced;FLAIR: Fluid-attenuated inversion recovery

*Progress is determined by any one project

\section{Materials And Methods}

Participant 
Retrospective analyses were performed on the Follow-up care of 64 adult patients with histologically

confirmed supratentorial LGGs (27 with astrocytoma, 22 with oligodendroglioma and 15 with oligoastrocytoma; WHO 2016 classification). Patients received their first surgery between May 2017 and November 2019, the same dose and course of radiotherapy and chemotherapy were given after operation(Radiotherapy,total dose:50.4 Gy,1.8 2.0 Gy/28 times[9,10];chemotherapy:75mg/m²,12/28 project,12 period.). After hospital they were regularly followed up by the neurosurgery group of the hospital. Tumor recurrence/progression was defined through clinical, radiological and/or metabolic evolution. Those who can not obtain pathological diagnosis should refer to the RANO criteria(see Table 1) and be judged by multi-disciplinary team discussion[11]. Metabolic assessments with positron emission tomography (PET) have previously been performed for the diagnosis and FU of patients with LGGs.

Ethical approval was obtained from our hospital Ethics Committee. The need for informing consent was waived due to the retrospective study. Patients included according the following criteria:1) Pathologically confirmed the patients with LGG;2)all patients underwent same chemotherapy and radiotherapy treatment after the prior surgery;3) tumor recurrence confirmed by imaging and clinical follow-up(follow-up time no less than 6 months). The exclusion criteria were as follows:1) patients with poor MRI images;2)patients with tumor hemorrhage;3) age < 18 years;4)patients with recurrent gliomas without same chemotherapy and radiotherapy et.al(the detail selective criteria were shown in Fig.1).Finally,a total of 64 patients with histologically confirmed glioma (WHO II) were collected for the study,among it, 22 patients were confirmed existing tumor recurrence(among it, 5 were confirmed by biopsy after secondary operation, 17 were considered according to RANO criteria.),some preoperative information like age,sex,FPS,IDH1,T1WI,T2WI-FLAIR and T1CE images were collected for analysis.

\section{Description of the region of interest (ROI)}

ITK-snap software (www.itksnap.org) was used for manual image segmentation. T1CE images were selected for ROI sketch, and the ROI delineation was carried out by two senior radiologists with more than 10 years of work experience using the double-blind method. In case of any disagreement, consensus was reached by discussion, especially on reading with a discrepancy.(see Fig.2:segmentation of ROI)

\section{Data preprocessing}

The dataset was randomly assigned in a 7:3 ratio to either the training set and validation set. All cases in the training set were used to train the predictive model, while cases in the validation set were used to independently evaluate the model's performance. Before analyses, variables with zero variance were not included in analyses. Then, the missing values were substituted for the median. Finally, the data were standardized by the z-score standardization[12].

\section{Feature selection}

All radiomic feature extraction was performed using MatLab 2016A(MathWorks, Natick, MA),396 features were extracted from each sequence, these features included the "Laplacian of Gaussian (LoG), rotation invariant local binary patterns (RILBP),the gray level co-occurrence matrix (GLCM), intensity-based features(IBF), directional Gabor texture features (DGTF), and rotation invariant circular Gabor features (RICGF) et al.Logistic regression were used for models construction based on the extracted optimal feature subsets of the training dataset.Feature screening was performed by using mRMR[13] methods alone or combines with univariate logistic analysis. The $P$ value of the univariate logistic analysis was 0.05 . Logistic-based radscore model was built based on the established optimal feature subsets of the training dataset.

\section{Development and validation of a radiomics nomogram}

The features with nonzero coefficients were combined to construct a formula that was used to calculate the

Rad-score for every glioma patient(The formula is shown in the supplementary Information). Rad-score of each patients and multivariable logistic regression analysis were used to establish the radiomics predictive models,and correspond nomogram was derived from the model.The nomogram was validated both in the training set and validation set. The ROC curve was performed to determine the performance of the radiomics models, accuracy, sensitivity, specificity and area under curve (AUC) were calculated. Calibration curves were used to evaluate these models and decision curve analysis(DCA)[14] were plotted to represent the clinical value of these models.Construction of model and nomogram was performed with $\mathrm{R}$ (version 4.0.2). A two-tailed $p$-value $<0.05$ indicated statistical significance.

\section{Statistical Analysis}

SPSS 22.0 software was used to compare the difference of age, gender, and other baseline characteristics between the training cohort and validation cohort were assessed respectively using the independent samples t-test, the $\chi^{2}$ test, and Mann-Whitney U-test.

\section{Result}

Among 64 patients, 22 were recurrent and another 42 cases were non-recurrence,age in recurrent group(RG) was higher than non-recurrence group(NRG), there was a significant difference between the two groups $(P<0.05)$.In the other clinicophathological characteristic,there were no significant between the RG and NRG,the result was showed in Table 2. 
The features screened from T1WI,T2WI-FLAIR,T1CE and multiparametric sequences were showed in Table 3,relied on the radiomics methods, four models were established for prediction LGG recurrence.Among them, in T1 sequence, 6 screened features were left to establish the model,it yielded 0.842 of AUC in training set and 0.79 in validation set;in T2 sequences, 5 features were left,the models yielded 0.785 of AUC in training set and 0.79 in the validation set;similarly in T1CE sequence, 4 features were left,the models yielded 0.784 and 0.803 of AUC in the training set and validation set respectively; the model composed of multi-parametric MRI was yielded the best performance among the four models in the study, 9 feature from T1,T2 and T1CE sequence were left, the AUC in training set and validation set were 0.966 and 0.93 respectively,the calibration curves of the model also indicated a good agreement between predicted probability and actual occurrence both in training set and validation set, these findings show that the nomogram was well calibrated, leading to high predictive performance.(The screened optimal features of models and the contributing weight of each modality or parametric were showed in Table 3, the performance of each models was showed in Table 4).

The DCA for the individual T1WI, T2WI-FLAIR, T1CE and the multi-parametric combination models are illustrated in Figure 5. Apparently, the net benefit of the model constructed based on the three sequences was higher than the individual sequence, to which it was superior across nearly the entire range of $P$ values.

Table 2. Baseline Demographics and Clinical Characteristics of Patients

\begin{tabular}{|c|c|c|c|c|}
\hline $\begin{array}{l}\text { Clinicophathological } \\
\text { characteristic }\end{array}$ & & Non-recurrence & Recurrence & $P$-value \\
\hline Numbers of cases & & 42 & 22 & \\
\hline age & & $40.60 \pm 12.20$ & $48.36 \pm 9.74$ & 0.047 \\
\hline \multirow[t]{2}{*}{ Sex,n(\%) } & 0 & $21(50)$ & $6(27)$ & \multirow[t]{2}{*}{0.291} \\
\hline & 1 & $21(50)$ & $16(73)$ & \\
\hline \multirow[t]{2}{*}{ IDH1-mutation,n(\%) } & 0 & $8(20)$ & $10(45)$ & \multirow[t]{2}{*}{0.128} \\
\hline & 1 & $34(80)$ & $12(55)$ & \\
\hline \multirow[t]{2}{*}{ Tumor crossing the midline, $\mathrm{n}(\%)$} & 0 & $26(63)$ & $12(55)$ & \multirow[t]{2}{*}{0.723} \\
\hline & 1 & $16(37)$ & $10(45)$ & \\
\hline Ki-67 & & $7.37 \pm 8.48$ & $6.18 \pm 4.47$ & 0.457 \\
\hline
\end{tabular}

Table 3.The features after screened of different modalities 


\begin{tabular}{|c|c|c|c|c|c|c|c|}
\hline modality & variables & Coef. & Std.Err. & $\mathrm{z}$ & $P>|z|$ & {$[0.025$} & 0.975] \\
\hline \multirow[t]{5}{*}{ T1CE } & intercept & -0.3825 & 0.5451 & -0.7017 & 0.4828 & -1.4508 & 0.6858 \\
\hline & GLCMEntropy_AllDirection_offset4_SD & 0.5540 & 0.5905 & 0.9382 & 0.3481 & -0.6034 & 1.7114 \\
\hline & Compactness2 & 0.6443 & 0.6101 & 1.0560 & 0.2910 & -0.5515 & 1.8401 \\
\hline & ShortRunEmphasis_AllDirection_offset7_SD & -1.1118 & 0.9869 & -1.1265 & 0.2600 & -3.0461 & 0.8226 \\
\hline & LongRunEmphasis_angle0_offset1 & -1.2584 & 0.7682 & -1.6382 & 0.1014 & -2.7640 & 0.2472 \\
\hline \multirow[t]{7}{*}{ T1WI } & intercept & 0.0196 & 0.3750 & 0.0523 & 0.9583 & -0.7154 & 0.7546 \\
\hline & GLCMEntropy_AllDirection_offset7_SD & 0.9189 & 1.1705 & 0.7850 & 0.4324 & -1.3753 & 3.2132 \\
\hline & LowGreyLevelRunEmphasis_AllDirection_offset1_SD & -3.9480 & 3.5082 & -1.1254 & 0.2604 & -10.8240 & 2.9279 \\
\hline & RunLengthNonuniformity_AllDirection_offset4_SD & 0.3930 & 0.4920 & 0.7987 & 0.4245 & -0.5714 & 1.3573 \\
\hline & ShortRunEmphasis_AllDirection_offset7_SD & -0.3207 & 0.4130 & -0.7765 & 0.4375 & -1.1302 & 0.4888 \\
\hline & ShortRunEmphasis_angle90_offset4 & 1.0657 & 2.3549 & 0.4525 & 0.6509 & -3.5498 & 5.6812 \\
\hline & Variance & 1.7391 & 0.7825 & 2.2225 & 0.0262 & 0.2054 & 3.2728 \\
\hline \multirow[t]{6}{*}{ T2WI-FLAIR } & intercept & -0.1973 & 0.4298 & -0.4591 & 0.6461 & -1.0397 & 0.6451 \\
\hline & ClusterShade_angle45_offset7 & 0.0706 & 0.3401 & 0.2075 & 0.8356 & -0.5960 & 0.7372 \\
\hline & Correlation_AllDirection_offset1_SD & -0.4181 & 0.4751 & -0.8800 & 0.3788 & -1.3494 & 0.5131 \\
\hline & Sphericity & -0.6831 & 0.4032 & -1.6941 & 0.0902 & -1.4734 & 0.1072 \\
\hline & HighIntensityLargeAreaEmphasis & -0.0726 & 0.4370 & -0.1662 & 0.8680 & -0.9291 & 0.7838 \\
\hline & LongRunEmphasis_angle90_offset1 & -1.4411 & 0.9134 & -1.5777 & 0.1146 & -3.2313 & 0.3491 \\
\hline \multirow[b]{10}{*}{ Multiparametric } & intercept & -0.1338 & 2.3732 & -0.0564 & 0.9550 & -4.7852 & 4.5176 \\
\hline & T1CE_ClusterProminence_angle90_offset7 & -2.4287 & 2.7045 & -0.8980 & 0.3692 & -7.7295 & 2.8721 \\
\hline & T1CE_InverseDifferenceMoment_AllDirection_offset7_SD & 2.3638 & 3.4494 & 0.6853 & 0.4932 & -4.3970 & 9.1245 \\
\hline & T2_GLCMEntropy_AllDirection_offset1_SD & 2.0994 & 2.7421 & 0.7656 & 0.4439 & -3.2750 & 7.4739 \\
\hline & T2_GLCMEntropy_AllDirection_offset4_SD & -1.2254 & 0.0745 & 1.683 & 0.0924 & -0.8635 & 1.5517 \\
\hline & T2_LongRunHighGreyLevelEmphasis_AllDirection_offset1_SD & 0.9696 & 1.2682 & 0.7646 & 0.4445 & -1.5161 & 3.4553 \\
\hline & T2_HaralickCorrelation_AllDirection_offset7_SD & -10.1476 & 6.6254 & -1.5316 & 0.1256 & -23.1332 & 2.8380 \\
\hline & T1_HaralickCorrelation_AllDirection_offset4_SD & -1.8642 & 1.6618 & -1.1218 & 0.2620 & -5.1213 & 1.3929 \\
\hline & T1_ShortRunEmphasis_AllDirection_offset7_SD & -1.9502 & 1.4283 & -1.3654 & 0.1721 & -4.7497 & 0.8493 \\
\hline & T1_HighIntensityLargeAreaEmphasis & 2.6325 & 1.6886 & 1.5590 & 0.1190 & -0.6770 & 5.9420 \\
\hline
\end{tabular}

Table 4.The performance of the models for predicting tumor recurrence in training set and testing set. 


\begin{tabular}{|c|c|c|c|c|c|c|c|}
\hline Modality & $\begin{array}{l}\text { Features screening } \\
\text { method }\end{array}$ & $\begin{array}{l}\text { Number of features after } \\
\text { screened }\end{array}$ & Cohort & $\mathrm{AUC}(95 \% \mathrm{Cl})$ & Sensitivity & Specificity & Accuracy \\
\hline \multirow[t]{2}{*}{ T1WI } & mRMR & 6 & Training & $\begin{array}{l}0.842(0.674- \\
0.905)\end{array}$ & 0.7 & 0.7 & 0.75 \\
\hline & & & Validation & $\begin{array}{l}0.79(0.687- \\
0.902)\end{array}$ & 0.778 & 0.778 & 0.78 \\
\hline \multirow[t]{2}{*}{ T2WI-FLAIR } & mRMR & 5 & Training & $\begin{array}{l}0.785(0.697- \\
0.912)\end{array}$ & 0.727 & 0.682 & 0.705 \\
\hline & & & Validation & $0.79(0.679-0.92)$ & 0.8 & 0.5 & 0.65 \\
\hline \multirow[t]{2}{*}{ T1CE } & mRMR & 4 & Training & $\begin{array}{l}0.784(0.665- \\
0.913)\end{array}$ & 0.889 & 0.556 & 0.722 \\
\hline & & & Validation & $\begin{array}{l}0.802(0.693- \\
0.911)\end{array}$ & 0.78 & 0.778 & 0.8 \\
\hline \multirow[t]{2}{*}{$\begin{array}{l}\text { Multi- } \\
\text { modalities }\end{array}$} & ULA+mRMR & 9 & Training & $\begin{array}{l}0.966(0.949- \\
0.99)\end{array}$ & 0.905 & 0.952 & 0.929 \\
\hline & & & Validation & $\begin{array}{l}0.93(0.905- \\
0.973)\end{array}$ & 1 & 0.8 & 0.90 \\
\hline
\end{tabular}

Abbreviation:UL,univariate logistic analysis.

\section{Discussion}

In our study,comparing the baseline characteristics between the RG and NRG,the age was the only factor that affect the recurrence,the result was consensus with a previous study: $\mathrm{Li}, \mathrm{J}$ et al.[8]developed a retrospective analysis of 84 patients with glioma that age was significantly correlated with the time of recurrence after first surgery,our result further indicated age was an important risk factor both affect the recurrence of LGG and high grade glioma(HGG).Jansen et al.[15]conducted a long-term follow-up of 110 patients with LGG after resection,single factor analysis demonstrated that the extent of resection were significant correlated with recurrence time of gliomas,the study of Patrizz, A.et al[16] indicated that patients with LGG after a resection, radiotherapy dose will significantly affect the survival time of patients.Although above studies showed some influenced factor about the recurrence of LGG, so far there is still lack of research that can quantitatively and accurately predict it after operation,therefore,our research developed a radiomics model that could predict the risk of recurrence before surgery.

Although histological analysis is the gold criteria for glioma grading, there is a main reason to illustrate that is not absolutely reliable: the tumor may contain high-grade lesions, pathological specimens resected during primary surgery could not completely reflect the nature of the whole tumor.Hence,relied on radiomics technology,we could extract numerous image parameters from the whole tumor region and quantitatively analyse the tumor properties.many previous studies have demonstrated that the application of radiomics technology can effectively differentiate high and low grade gliomas[17-18];Quan,Z et al.[19]used 51 LGG patients and developed the radiomics model to differentiate recurrence from radiation necrosis in gliomas, these previous studies could provide reference for strategy determine.In our study,64 LGG cases were used to establish radiomics models and also yield an optimal performance.It is conducive to clinician to develop more accurate treatment plans and prognosis evaluation for patients with high risk of recurrence.

So far,surgical resection, postoperative radiotherapy and chemotherapy are the basic methods for glioma treatment, Patrizz A et al.[16] indicated that histopathologic findings following chemoradiation do not correlate with clinical outcomes,therefore,all the cases in the study were underwent a radiotherapy and chemotherapy. In addition,high-level evidence-based medical evidence is lacking for the survival benefit of patients with recurrent gliomas,patients with a recurrent tumor undergoing a secondary surgery are few, hence, it is not easy to determine the nature of recurrent gliomas by biopsy. In our study,we estimated post-operative recurrence gliomas according to RANO criteria[11,20] and finally determination by a multidisciplinary discussion.

When numerous image features were extracted from each sequence but sample size is relatively small, over-fitting[21] is easily emerge,for avoiding over-fitting,mRMR were used for feature dimensionality reduction, features can be selected using mutual information, correlation or distance/similarity scores,it is an efficient data screening tool that has been widely used in several studies[22-23].All these 9 features of the combination model are texture features,which is an important visual feature and it is generally used to describe the spatial arrangement of intensities of images. The research of Qian T et al.[17] proved that texture features has the best predictive efficiency than other radiomics features in discriminating the high and low grade gilomas.

Studies have proven that some molecular alterations show great predictive and prognostic value in gliomas recurrence,numerous studies have shown that in grade II III gliomas ,1p/19q chromosomal codeletion is associated with improved PFS and OS.Similarly, IDH mutations are a good prognostic marker for OS improvement in grade II III glioma[19,24]. However, it's not satisfying to predict the recurrence of LGG only based on genotype alone[15].More accurate predictions require us to develop.Therefore,base on the MRI technology and numerous image features,we developed a radiomics methods to discriminate the recurrence LGG and non-recurrence LGG.According to our study $ه$ the combination model of multi-parametric MRI 
features yield a AUC of 0.966 in testing set and 0.93 in validation set,these results were obviously better than the individual MRI sequence model;in addition, the DCA showed that the net benefit of using a radiomics nomogram constructed on the basis of all three sequences was higher than any single sequence, it demonstrated that the nomogram we constructed is worthy of clinical use.

There are also some limitation in the study:1)according to Central Nervous System Cancers, Version 3.2020, National Comprehensive Cancer Network (NCCN), Clinical Practice Guidelines in Oncology[25],there is a lack of high-level evidence-based medical evidence for the survival benefits of secondary surgery in patients with recurrent glioma,so the major patients with glioma recurrence after surgery choose radiotherapy and chemotherapy in our institution,it means the recurrent tumor biopsy is difficult to acquire, RANO criteria is applied to selected case,but the total sample is still small,longer time and more available cases will be conducted in the future studies;2)Variety life habits, mental state and other factors that may affect tumor recurrence can not be excluded, which is also a difficulty encountered in many studies;3)so far there is few studies and still difficult to illustrate the relationship between the image feature and pathology of tumor,it deserves further study.

In conclusion,the appliance of radiomics methods can efficiently evaluate the risk of recurrence of LGG after the first surgery,our nomogram could provide a simple and direct predictive tool for clinician.

\section{Declarations}

Ethics approval and consent to participate

Ethical approval was obtained from the institutional Human Research Ethics Committee. The need for informed consent was waived due to the retrospective nature of the study.

Consent for publication

Yes

Availability of data and materials

The data will be available via connecting the corresponding author.

Competing interests

The authors declare that they have no conflict of interest.

Funding

This work was supported and funder by the Key R \& D Projects in Jiangxi Province,China,(NO.20171ACG70002).

Acknowledgements

No apply

Authors' contributions:

Conceptualization: Zhenhua Wang, Xinlan Xiao, Ken He, Peipei Pang

Data curation: Zhenhua Wang, Zhaotao Zhang

Formal analysis: Zhenhua Wang, Xinlan Xiao, Ken He, Feng Hu

Funding acquisition: Zhenhua Wang, Xinlan Xiao, Ken He, Di Wu

Investigation: Zhenhua Wang, Xinlan Xiao

Methodology: Zhenhua Wang, Xinlan Xiao

Project administration: Zhenhua Wang, Xinlan Xiao, Ken He, Peipei Pang,

Resources: Zhenhua Wang, Xinlan Xiao, Ken He,Peipei Pang

Software: Zhenhua Wang, Xinlan Xiao, Peipei Pang

Supervision: Zhenhua Wang, Xinlan Xiao

Validation: Zhenhua Wang, Xinlan Xiao

Visualization: Zhenhua Wang, Xinlan Xiao, Peipei Pang

Page 7/12 
Writing - original draft: Zhenhua Wang, Xinlan Xiao, Peipei Pang

Writing - review \& editing: Zhenhua Wang, Xinlan Xiao, Peipei Pang

\section{References}

[1]Ganau L, Paris M, Ligarotti G, et al. Management of gliomas: overview of the latest technological advancements and related behavioral drawbacks.Behav Neurol. 2015;2015:862634.

[2]Tully PA, Gogos AJ, Love C, et al. Reoperation for recurrent glioblastoma and its association with survival benefit. Neurosurgery. 2016;79:678-689.

[3]Ostrom Q, Gittleman H, Fulop J, et al. CBTRUS statistical report: primary brain and central nervous system tumors diagnosed in the United States in 2008-2012. Neuro Oncol. 2015;17(Suppl 4):iv1-iv62.

[4]Reavey-Cantwell J, Haroun R, Zahurak M, et al. The prognostic value of tumor markers in patients with glioblastoma multiforme: analysis of 32 patients and review of the literature. J Neurooncol. 2001;55:195-204.

[5]Westphal M, Giese A. Local control of gliomas:the next best step-a good step? Front Radiat Ther Oncol. 1999;33:214-226.

[6]De Fazio S, Russo E, Ammendola M, et al. Efficacy and safety of bevacizumab in glioblastomas.Curr Med Chem. 2012;19:972-981.

[7]Tugcu B, Postalci L, Gunaldi O, et al. Efficacy of clinical prognostic factors on survival in patients with glioblastoma. Turk Neurosurg. 2010;20:117125.

[8]Li, J., Niu, X., Gan, Y., Yang, Y., Wang, T., Zhang, H., Liu, Y., \& Mao, Q. (2019). Clinical and Pathologic Features and Prognostic Factors for Recurrent Gliomas. World neurosurgery, 128, e21-e30.

[9]Shaw E,Arusell R,Scheithauer B,et al. Prospective randomized trial of low-versus high-dose radiation therapy in adults with supratentorial low-grade glioma:initial report of a North Central Cancer Treatment Group/Radiation Therapy Oncology Group/Eastern Cooperative Oncology Group study [J].J Clin Oncol,2 00 2,2 0(9):2 26 7-2 276.

[10]Karim AB,Maat B,Hatlevoll R,et al.A randomized trial on dose-response in radiation therapy of low-grade cerebral glioma:European Organization for Research and Treatment of Cancer(EORTC)Study 22 844[J].Int J Radiat Oncol Biol Phys,1996,3 6(3):5 49-556.

[11]Yamanaka T,Shirota S,Sawai Y,et al.Osteoporosis as a risk factor for the recurrence of benign paroxysmal positional vertigo[J].Laryngoscope,2013,123(11):2813-2816.

[12]Kranzusch R, Aus dem Siepen F, Wiesemann S, et al. Z-score mapping for standardized analysis and reporting of cardiovascular magnetic resonance modified Look-Locker inversion recovery (MOLLI) T1 data: Normal behavior and validation in patients with amyloidosis. J Cardiovasc Magn Reson. 2020;22(1):6. Published 2020 Jan 20. doi:10.1186/s12968-019-0595-7

[13]Peng H, Long F, Ding C. Feature selection based on mutual information: criteria of max-dependency, max-relevance, and min-redundancy. IEEE Trans Pattern Anal Mach Intell. 2005;27(8):1226-1238.

[14]Vickers AJ, Elkin EB. Decision curve analysis: a novel method for evaluating prediction models. Med Decis Making 2006;26:565-574.

[15]Jansen E,Hamisch C,Ruess D,et al.Observation after surgery for low grade glioma:long-term outcome in the light of the 2016 WHO classification[J].J Neurooncol,2 01 9,1 45 (3):5 0 1-5 07.

[16]Patrizz, A., Dono, A., Zhu, P., Tandon, N., Ballester, L. Y., \& Esquenazi, Y. (2021). Tumor recurrence or treatment-related changes following chemoradiation in patients with glioblastoma: does pathology predict outcomes?. Journal of neuro-oncology, 10.1007/s11060-020-03690-7.

[17]Tian, Q., Yan, L. F., Zhang, X., Zhang, X., Hu, Y. C., Han, Y., Liu, Z. C., Nan, H. Y., Sun, Q., Sun, Y. Z., Yang, Y., Yu, Y., Zhang, J., Hu, B., Xiao, G., Chen, P., Tian, S., Xu, J., Wang, W., \& Cui, G. B. (2018). Radiomics strategy for glioma grading using texture features from multiparametric MRI. J Magn Reson Imaging, 48(6), 1518-1528.

[18]Wang, Q., Li, Q., Mi, R., Ye, H., Zhang, H., Chen, B., Li, Y., Huang, G., \& Xia, J. (2019). Radiomics Nomogram Building From Multiparametric MRI to Predict Grade in Patients With Glioma: A Cohort Study. Journal of magnetic resonance imaging : JMRI, 49(3), 825-833.

[19]Zhang, Q., Cao, J., Zhang, J., Bu, J., Yu, Y., Tan, Y., Feng, Q., \& Huang, M. (2019). Differentiation of Recurrence from Radiation Necrosis in Gliomas Based on the Radiomics of Combinational Features and Multimodality MRI Images. Computational and mathematical methods in medicine, 2019, 2893043.

[20]Chukwueke, U. N., \& Wen, P. Y. (2019). Use of the Response Assessment in Neuro-Oncology (RANO) criteria in clinical trials and clinical practice. CNS oncology, 8(1), CNS28. https://doi.org/10.2217/cns-2018-0007 
[21]Balagurunathan Y, Kumar V, Gu Y, et al. Test-retest reproducibility analysis of lung CT image features. J Digit Imaging 2014;27:805-823.

[22] Cai Y, Huang T, Hu L, Shi X, Xie L, Li Y. Prediction of lysine ubiquitination with mRMR feature selection and analysis. Amino Acids. 2012;42(4):1387-95.

[23]. Alshamlan H, Badr G, Alohali Y. mRMR-ABC: a hybrid gene selection algorithm for Cancer classification using micro-array gene expression profiling. Biomed Res Int. 2015;2015:604910.

[24]Jiang H,Cui Y,Wang J,et al.Impact of epidemiological characteristics of supratentorial gliomas in adults brought about by the 2016 world health organization classification of tumors of the central nervous system[J].Oncotarget,2017,8 (1 2):20354-20361.

[25]Nabors LB, Portnow J, Ahluwalia M, Baehring J, Brem H, Brem S, Butowski N, Campian JL, Clark SW, Fabiano AJ, Forsyth P, Hattangadi-Gluth J, Holdhoff M, Horbinski C, Junck L, Kaley T, Kumthekar P, Loeffler JS, Mrugala MM, Nagpal S, Pandey M, Parney I, Peters K, Puduvalli VK, Robins I, Rockhill J, Rusthoven C, Shonka N, Shrieve DC, Swinnen LJ, Weiss S, Wen PY, Willmarth NE, Bergman MA, Darlow SD. Central Nervous System Cancers, Version 3.2020, NCCN Clinical Practice Guidelines in Oncology. J Natl Compr Canc Netw. 2020 Nov 2;18(11):1537-1570.

\section{Figures}

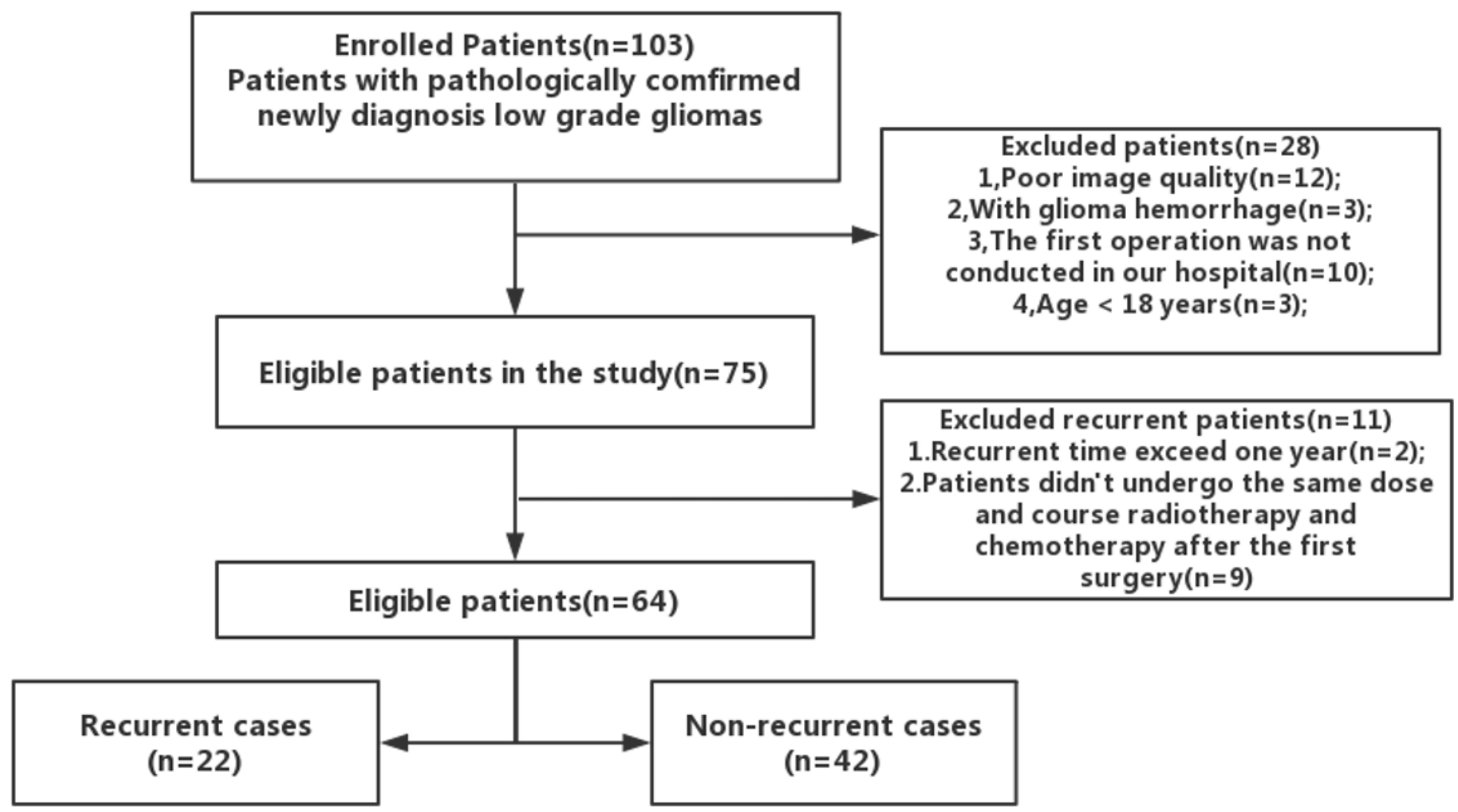

Figure 1

flow diagram for patient selection 

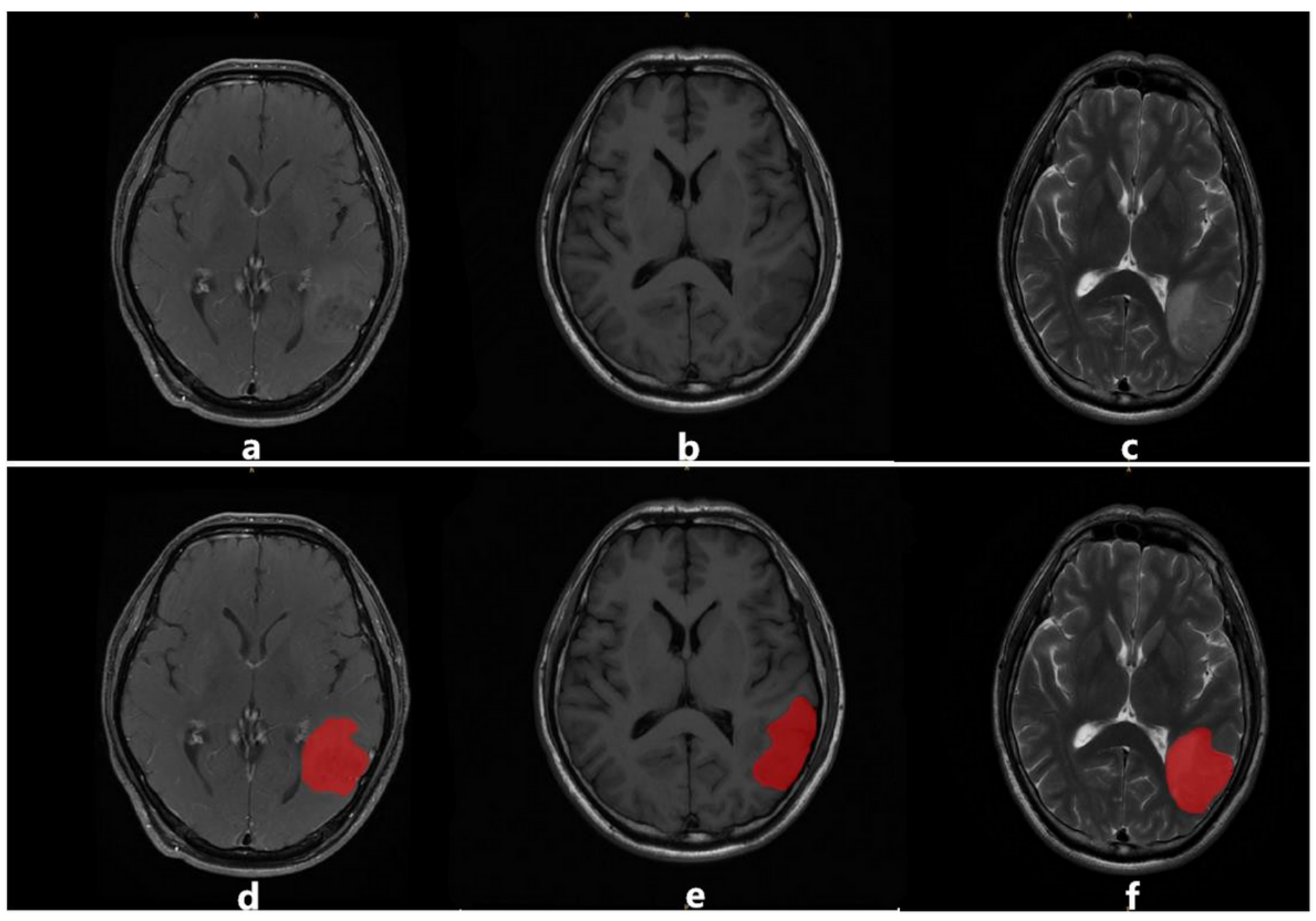

Figure 2

An example of image segmentation: a,b and c were T1CE,T1WI and T2WI-FLAIR image respectively; $d$,e and f represented the region of tumor(red area),these region will serve as region of interest(ROI )for feature extraction. 


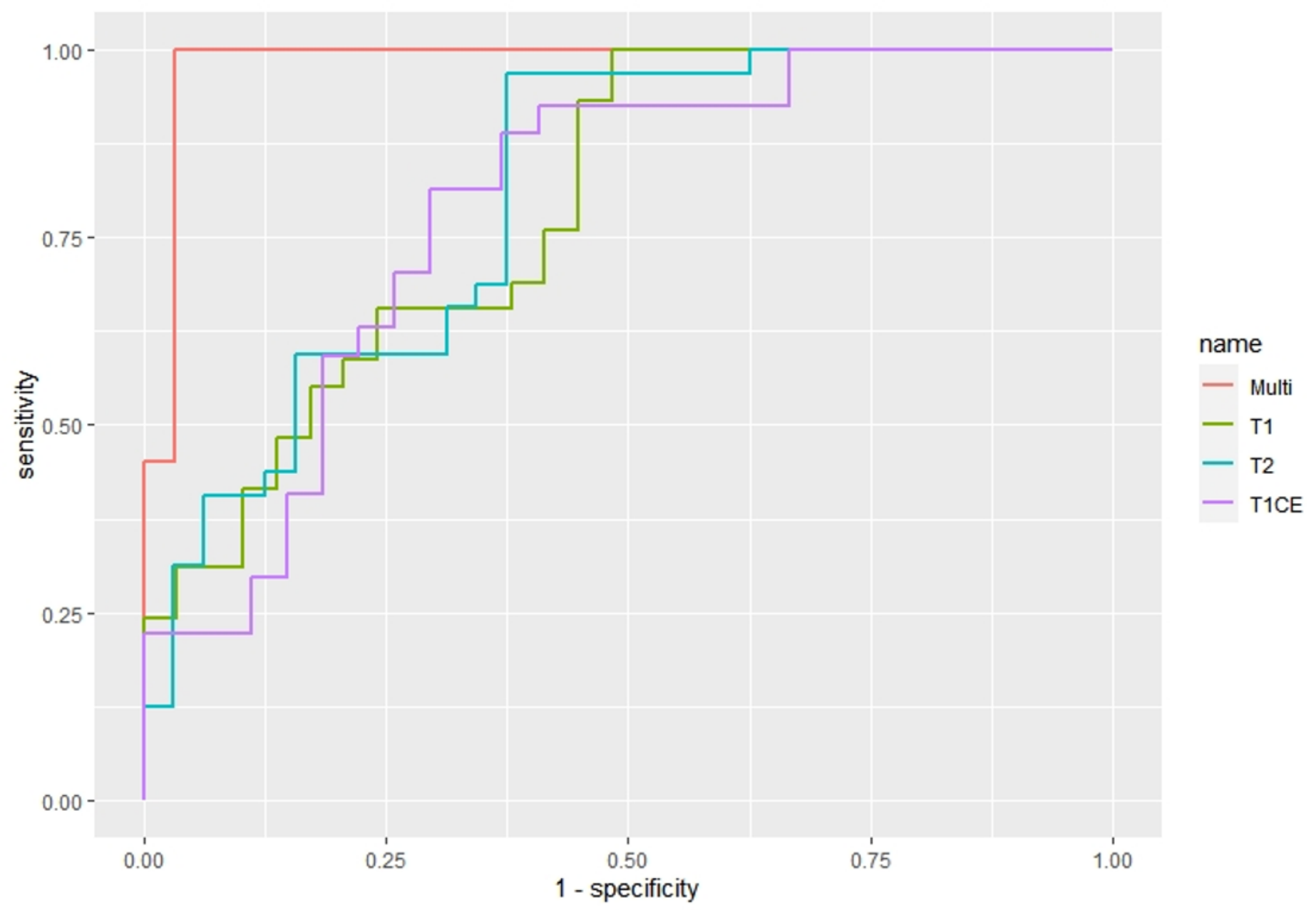

Figure 3

The ROC curve of 4 sorts of prediction models: red curve represents the model construct by the multiparametric MRI sequences(T1WI,T2WI-FLAIR and T1CE), the green curve represents model of T1WI,the blue curve represents T2WI-FLAIR and the purple curve represents T1CE.

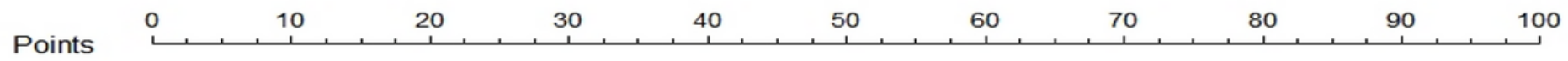

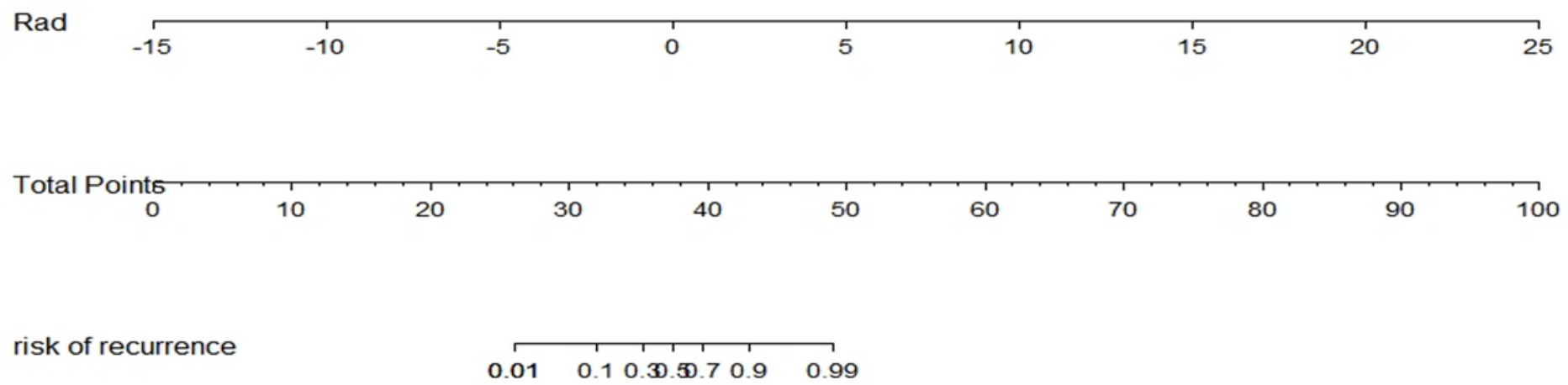

\section{Figure 4}

The nomogram was developed by the combination of T1WI,T2WI-FLAIR and T1CE image features. 

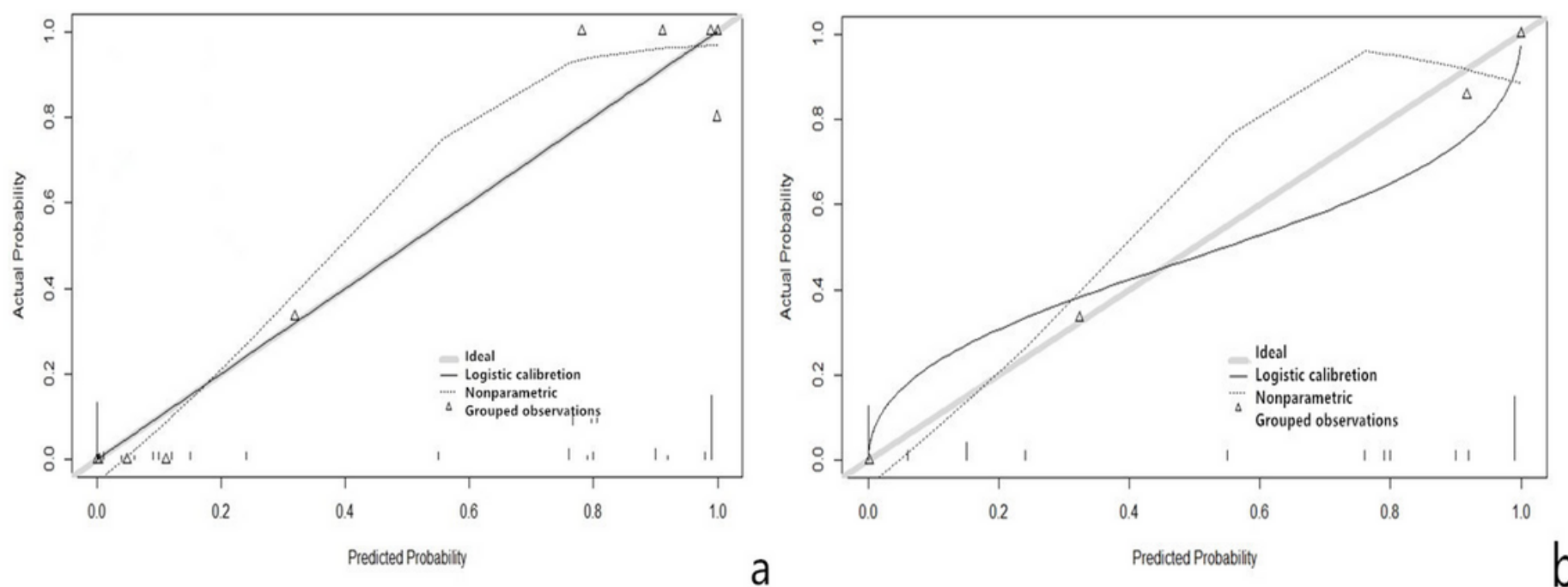

Figure 5

$(a, b)$ :Performance of the nomogram for predicting the recurrence of LGG, The $y$-axis represents the actual probability and the $x$-axis represents the predicted probability, Fig.a show the model's calibration of training set and Fig.b show those of validation set. A calibration curve describes the consistency between the predicted and actual incidence rate of recurrence, $45^{\circ}$ gray heavy lines represent ideal prediction performance, non- $45^{\circ} \mathrm{dotted}$ lines represent the prediction performance of the nomogram model, and non- $45^{\circ}$ solid lines represent the correction of the prediction performance deviation of the model. The closer the solid line is to the ideal gray line, the better the prediction accuracy of the nomogram is.

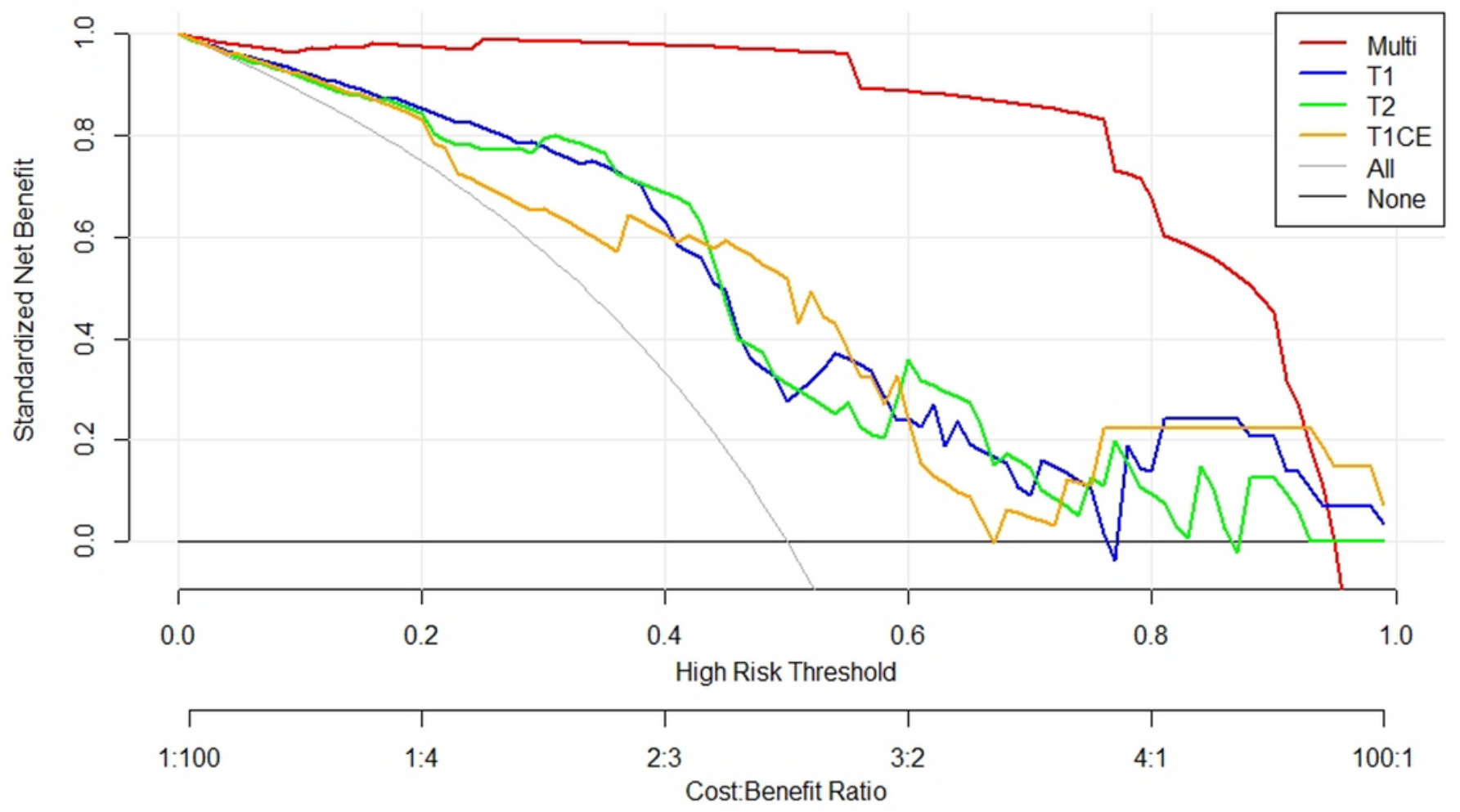

Figure 6

Decision curve analysis for the models were established using the radiomics features from T1WI,T2WI-FLAIR and T1CE sequences. The $y$-axis measures the net benefit. The blue, green and yellow curves represent the models based on T1WI, T2WI-FLAIR, and T1CE sequence respectively. The red curve represented the model based on the combination of the three sequences. The gray curve represents the assumption that all patients were treated and the straight black line at the bottom of the figure represents the assumption that none patients were treated. 